\title{
Frequency Estimation of Weighted Signals Based On DFT Interpolation Algorithm
}

\author{
Chang-Gui Xie \\ Chongqing Vocational Institute of Engineering \\ Chongqing, 402260, People's Republic of China \\ xiechanggui_125@sina.com
}

\begin{abstract}
It is generally demanded for strict frequency estimation of sinusoidal signal in a good many engineering practice situations. This paper puts forward an iterative frequency estimation algorithm on strength of the interpolation of Fourier coefficients of weighted samples. The algorithm is practically believed to be almost applicative for all regular window functions. Systematic errors of a variety of windows are given in this paper, and the proposed algorithm's performance is examined and verified with occurrence of white Gaussian noise. The outcomes of simulation identify that it could be significantly lessened for errors touched off by the mistaken location of spectral line. It is direct and candid to implement the proposed algorithm with high precision, benign compatibility and favorable robustness to additive noise, which altogether endow it a perfect algorithm to strictly estimate the frequency of spectral analysis.
\end{abstract}

Keywords-Discrete Fourier Transform; Normalized frequency; Parameter estimation; Zero-padding

\section{INTRODUCTION}

Accurate parameters estimation (frequency, amplitude, and phase) of sinusoids contaminated with random noise appears in subject of various fields for several decades. For instance, a typical problem encountered in vibration analysis of rotating machinery is to estimate parameters of a sampled multi-frequency signals under additive noise. Usually, that a new peak value emerges in spectrum or the root-mean-square (RMS) value of the vibration at an integer multiple of the fundamental frequency changes, can be regarded as the sign of a probable error [1]. With the increasing application of non-linear devices and periodical time-variable loads in electrical power system, distortion of current and voltage waveforms becomes a serious problem. Therefore, a real-time analysis and control of electric power harmonic is of great significance for maintaining the electric energy quality, preventing damage to the electric network systems and saving energy [2-3]. Besides, a number of audio coding technologies have been developed recently, in which the audio signal is decomposed into sinusoids and noise before coding. The decomposition, of coures, depends on the accurate frequency estimation of audio signal. Previous literature has introduced various estimation approaches which can be generally classified into time domain and frequency domain ones. Attributing to the easy operation and high efficiency, the frequency domain approaches, based on the discrete Fourier transform (DFT) and implemented by the fast Fourier transform (FFT), are often used. However, there are also some inherent drawbacks in frequency domain methods, such as the picket fence effect and the spectral leakage effect, which will bring about significant errors in frequency estimates if the signal is noncoherently sampled $[4,5]$. Studies has shown that if we can afford additional computational cost, it is possible to compensate the errors and obtain high-accuracy frequency estimates even with a small number of samples.

The interpolated DFT algorithm (IpFA) is one of the most widely studied method to correct the errors. The essence of this method is to obtain the frequency estimate of a signal with the weighted average of a certain number of known spectral bins [6-8]. When a maximum sidelobe decay window is selected, the frequency can be estimated by means of simple analytical relationships. Based on the research of Offelli and Petri, Duda deduced the polynomial approximation interpolation algorithm (which is suitable for the Dolph-Chebyshev windows and the Kasier-Beaasel windows [12]. Nevertheless, the polynomial coefficients are demanded to be calculated before analysis, which signifies that the coefficients of various windows must be worked out and saved in memorizer in advance. The process is inevitably intensive and troublesome, particularly for the adjustable windows whose properties can be regulated by one or multiple parameters.

In this paper, we firstly proposed a new interpolation algorithm for the Hanning window, in which the zero padding technique was adopted to obtain more spectrum lines within a frequncy interval. Subsequently, the new algorithm was extended to be compatible with other classic windows by introducing the main-lobe fitting technique $[9-11,15]$. We studied the systematic errors of the suggested method for various windows as well as the performance under white Gaussian noise. Finally, a comparative study was done. It was demostrated by simulation that in noise condition the proposed algorithm was more robust than the traditional algorithms.

\section{THEORETICAL BACKGROUND}

Let us consider, for simplicity but without loss of generality, the continuous cosine signal contaminated with additive white noise, which is given in the form 


$$
y(t)=A \cos \left(2 \pi f_{0}+\varphi_{0}\right)+z(t),
$$

where $A$ denotes the amplitude, $f_{0}$ denotes frequency, $\varphi_{0}$ denotes the phase angle, $t$ denotes the continuous-time variable and $z(t)$ is the white noise. After sampling at frequency $f_{\mathrm{s}}$ over the observation interval $N \Delta t\left(\Delta t=1 / f_{s}\right)$, the following discrete cosine signal of $N$ samples

$$
y(n)=A \cos \left(2 \pi \frac{f_{0}}{f_{s}} n+\varphi_{0}\right)+z(n), n=0,1 \cdots N-1,
$$

becomes available. To meet the Ny-quist Sampling Theorem, it is supposed that $f_{s}$ surpasses $2 f_{0}$. The frequency resolution is obtained by $\Delta f=f_{s} / N$. If the signal is asynchronously sample

the normalized frequency ${ }^{\psi_{0}}$ lies between two largest spectral lines[13].Therefore, $\psi_{0}$ can be further written as:

$$
\psi_{0}=\beta_{w}+\mu_{w}
$$

where $\beta_{w}$ and $\mu_{w}\left(-0.5<\mu_{w} \leq 0.5\right)$ are respectively the integer part and the fractional part. $\psi_{0}$ could be also defined as

$$
\frac{f_{0}}{f_{s}}=\frac{\psi_{0}}{N}
$$

The integer part $\beta_{w}$ can be readily and correctly determined by means of a maximum search routine, as long as the SNR is above threshold. The weighted samples can be acquired when signal samples $y(n)$ is multiplied by data window values $w(n)$ :

$$
y_{w}(n)=A \cos \left(2 \pi \psi_{0} n+\varphi_{0}\right) w(n),
$$

On strength of the definition of DFT, the weighted signal $y_{w}(n)$ of DFT coefficients can obtain calculated as:

$$
Y_{w}(k)=\frac{A}{2} e^{j \varphi_{0}} W_{N}\left(k-\psi_{0}\right)+\frac{A}{2} e^{-j \varphi_{0}} W_{N}\left(k+\psi_{0}\right),(6)
$$

In the above Equation (6), $W_{N}(k)$ represents the window $w(n)$ from Discrete Time Fourier Transform (DTFT). Putting equation (3) into (6), the largest magnitude would be acquired through:

$$
\left|Y_{w}\left(\beta_{w}\right)\right|=\frac{A}{2}\left|e^{j \varphi_{0}} W_{N}\left(-\mu_{w}\right)+e^{j \varphi_{0}} W_{N}\left(2 \beta_{w}+\mu_{w}\right)\right| . \text { (7) }
$$

In equation (7), the second sign on the right side means leakage offer by imaginary part of the spectrum. The conditions are postulated that, $\psi_{0}$ meets the requirements of $\psi_{0}>5$ and $\psi_{0}<N / 2-5$ so as to make sure leakage cased by the spectrum's negative part is the smallest which could be neglected.

Similarly, the second and the third largest spectral lines can also be determined. With proper combination of two or more spectral lines, a ratio $\chi$ can be obtained which only depends on the selected window and the frequency deviation $\mu_{w}$. In other words, if the data window is known, $\mu_{w}$ can be solely determined by $\chi$

$$
\mu_{w}=h(\chi)
$$

For maximum side-lobe decay windows $[2,3,5,7,8$, $13,14], \mu_{w}$ can be written as a function of $\chi$ in a simple and explicit form. For others, the polynomial approximation solution is suggested. In next part, we will introduce an interpolation algorithm which is simple and can be applied to almost all classic windows.

\section{Proposed Algorithm}

\section{A. Proposed Algorithm}

Zero-padding is a technique defined as appending zero values to the weighted samples prior to the DFT calculation. The appended zero values are treated as additional samples collected at the same rate, and therefore extending the measurement time, as shown in (8)

$$
y_{a z}(n)=\left\{\begin{array}{l}
y_{w}(n), 0 \leq n \leq N \\
0, N \leq n \leq L
\end{array},\right.
$$

where $L-N$ zeros are added. Acoordingly, the discrete spectrum extends as well. It results in more accurate sampling of the signal spectrum because, instead of $N$ spectrum samples, $M \cdot N$ samples of the same spectrum are available. However, it shoud be pointed out that padding the data with zeros and computing a longer FFT does give more frequency domain points, but it does not improve the fundamental limit, nor does it alter the effects of aliasing error[14]. The resolution limits are established by the observation interval and the sampling rate. No amount of zero padding can improve these basic limits and the spectrum parameters, such as signal-to-noise ratio and the spectrum leakage level, remain unchanged.The DFT of Eq.(9) by 


$$
Y(k)=\sum_{n=0}^{N-1} y_{a z}(n) e^{-j \frac{2 \pi k}{N} \frac{k}{\lambda} n} .
$$

Compared with Eq.(4), we obtain that

$$
Y(k)=Y_{w}\left(\frac{k}{\lambda}\right)
$$

Acoordingly, Eq. (7) can be reformulated as

$$
|Y(\beta)|=\frac{A}{2}\left|e^{j \varphi_{0}} W_{N}(-\mu)+e^{-j \varphi_{0}} W_{N}\left(\frac{2 \beta}{\lambda}+\mu\right)\right|,
$$

where $\beta$ and $\mu$ are respectively the integer part and the fractional part of $\lambda \psi_{0}$. Similarly, $\beta$ is retruned by the maximum search routine of $Y(k)$. At this stage, three largest lines of $Y(k)$ are given by $|Y(\beta)|,|Y(\beta+1)|$ and $|Y(\beta-1)|$.

The second term on the right in (12) represents the contribution from the imaginary part in the spectrum. If $\psi_{0}$ satisfies that $5<\psi_{0}<N / 2-5$, the interference form the second termcan will be very small and can be neglected. With this assumption, (12) reduces to

$$
|Y(\beta)| \cong \frac{A}{2}\left|W_{N}(-\mu)\right|
$$

Similarly, we have that $|Y(\beta+1)| \cong \frac{A}{2}\left|W_{N}(\sigma-\mu)\right|$. and

$|Y(\beta-1)| \cong \frac{A}{2}\left|W_{N}(\sigma+\mu)\right| .$, where $\sigma=\frac{1}{\lambda}$. In particular, for the Hanning window we have $\left|W_{N}(k)\right| \cong \sin (\pi k) / h(k), h(k)=2 \pi k$.

$$
|Y(\beta)| \cong \frac{A}{2} \frac{\sin (\pi \mu)}{h(\mu)},
$$

$$
|Y(\beta+1)| \cong \frac{A}{2} \frac{\sin \pi(\sigma-\mu)}{h(\sigma-\mu)},
$$

$$
|Y(\beta-1)| \cong \frac{A}{2} \frac{\sin \pi(\sigma+\mu)}{h(\sigma+\mu)}
$$

Expanding the sine terms in Eqs. (14b) and (14c) and combining them together, we achive that

We now introduce two variables $\varepsilon_{1}, \varepsilon_{2}$ defined as

$$
\begin{aligned}
\varepsilon_{1} & =\frac{|Y(\beta-1)|}{|Y(\beta)|}, \\
\text { and } \varepsilon_{2} & =\frac{|Y(\beta+1)|}{|Y(\beta)|} .
\end{aligned}
$$

For simplicity and conciseness, in the following parts,

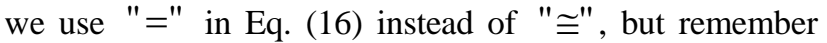
that the approximation relationship still remains. Now, Eq. (16) can be rewritten as

$$
h(\mu+\sigma) \varepsilon_{1}+h(\mu-\sigma) \varepsilon_{2} \cong 2 h(\mu) \cos (\pi \sigma) .
$$

Expanding the terms $h(\sigma+\mu), h(\mu-\sigma)$ and $h(\mu)$, and finally the value of $\mu$ could be obtained as

$$
\mu=\frac{\sigma\left(\gamma_{1}-\gamma_{2}\right)}{\cos (\pi \varepsilon)-\gamma_{1}-\gamma_{2}}
$$

Because the value belong to interval $(0.5,0.5]$,so eventually the decimal part of line frequency correction can be obtained as follow:

$$
\hat{\mu}=\frac{\sigma\left(\gamma_{1}-\gamma_{2}\right)}{\cos (\pi \varepsilon)-\gamma_{1}-\gamma_{2}}
$$

$$
h(\sigma+\mu)|Y(\beta-1)|+h(\sigma-\mu)|Y(\beta+1)| \cong 2|Y(\beta)| h(\mu) \cos (\pi \sigma)
$$




\section{B. Extension for Other Classic Windows with Mainlobe Fitting}

In Subsection above, we have deduce the interpolation for the Hanning window with zero padding. In this subsection, it will be extended for other classic windows with mainlobe fitting technique. Set

$$
X(k)=\left[W_{O}(k)\right]^{p}+W_{H}(k),
$$

where $W_{O}(k)$ denotes the normalized spectrum of any classic window, $W_{H}(k)$ denotes the normalized spectrum of the Hanning window, $p=X L_{H a n} / X L_{w(n)}$. $X L$ is the scalloping loss (SL) of the Hanning window and the corresponding window selected in (21), respectively. Scalloping loss is defined as the ratio of coherent gain for a frequency component located halfway between DFT bins to the coherent gain for a frequency component located exactly at a DFT bin, as indicated as

$$
S L=X L=\frac{W(0.5)}{W(0)}
$$

where $W(\bullet)$ is the DTFT of the relevant window. It can be found that $|S(k)|$ is very small for most classic winodws if $k$ is in the range $(-0.5,0.5]$. For example, the maximum value of $|S(k)|$ is less than $10^{-5}$ for the Hamming window and less than $10^{-4}$ for the Blackman window. That means $\left[W_{O}(k)\right]^{p}$ and $W_{H}(k)$ fits well with each other in the middle of their mainlobes.[16] It is implied that the above interpolation algorithm can be extended for the classic window as long as $k$ is limited in the range of $[-0.5,0.5]$. With zero padding technique $(\lambda \geq 3)$, it is easy for the three largest spectral lines, which are used in the interpolation algorithm, to meet the requirement. Given the amount of computation and the radix-2 FFT algorithm, we often choose $\lambda=4$ in practice mesaurement.

\section{COMPARATIVE STUDY}

In order to assess the proposed algorithm's effectiveness, the proposed research is tested compaired with some traditional research methods in the Matlab simulation environment. This article chose two interpolation algorithm of Hanning window proposed by Grandke in 1983[9], the energy centrobaric method proposed by Dingkang in 1996[5], three polynomial approximation interpolation algorithm proposed by Duda in 2011[12], the average interpolation algorithm proposed by Belega in 2013 and other traditional methods [11]. In the simulation test, the new algorithm's correction precision of frequency was tested compared with the traditional algorithm under the noise condition.

\section{A. Study of Noise Influence}

Before simulation analysis, the normalized frequency deviation was set in the interval of $(-0.5,0.5)$, with the scanning step of $0.05 \mathrm{HZ}$, the sampling frequency of $1024 \mathrm{HZ}$, also accompanied by the 1024 sampling points. This was done in order to better evaluate the ability to estimate the frequency deviation of each algorithm. For the above selected frequency deviation range, the simulation results are shown in Figure 1.

Figure 1 shows that for four kinds of algorithms including the new algorithm, the maximum absolute value of the selected frequency between the real value and the estimated value. Each algorithm was all weighted in the Hanning window before the DFT operation began. As shown in Figure 1, except for D.Kang and Belege, the rest of the above-described four kinds of algorithms all received a high estimation accuracy,and Duda's worked out the best.

Additionally, the results of weighted samples of each algorithm in Hamming window and Blackman window were displayed. As XieMing could only be weighted in Hanning window, Figure 2 and Figure 3 give the simulation results of the remaining four kinds of algorithm.

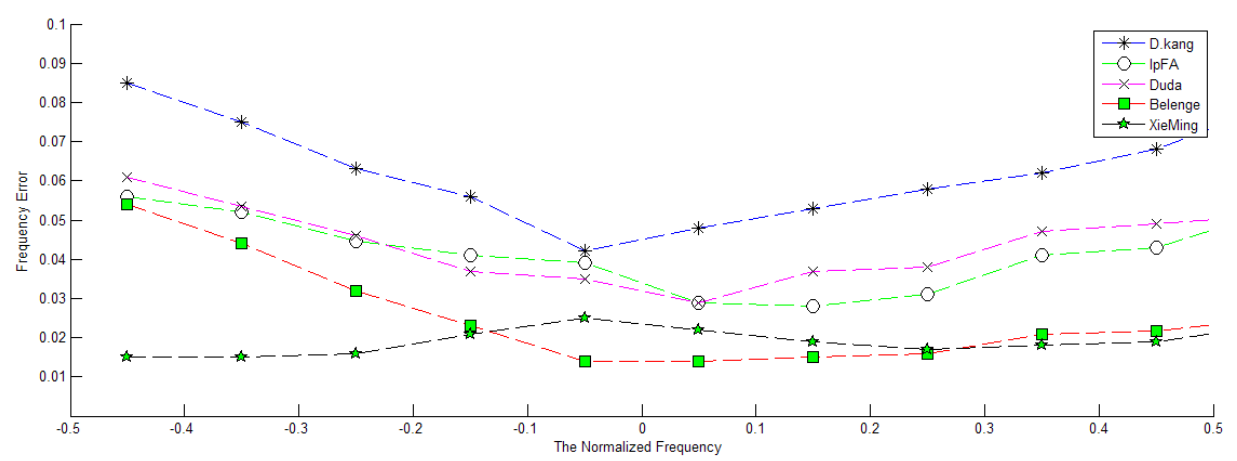

Figure 1. Frequency Error 


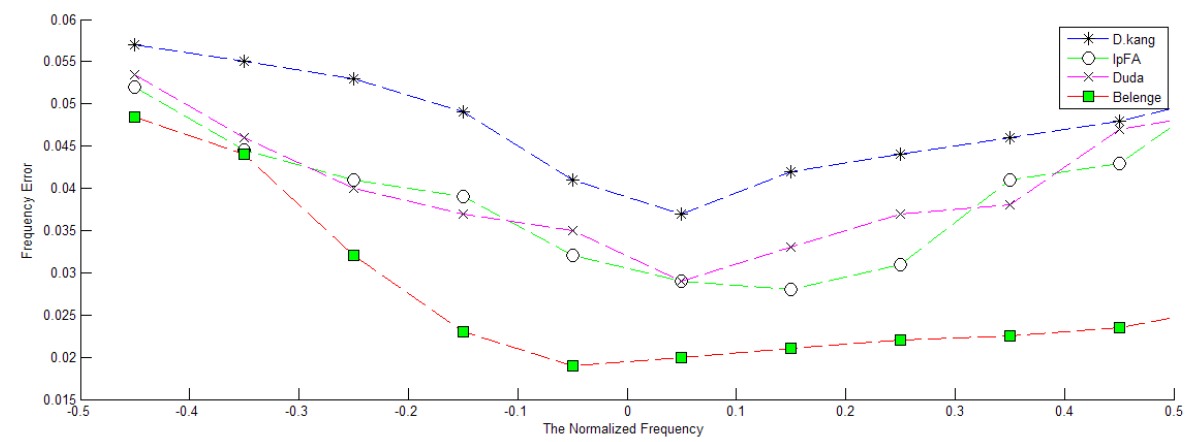

Figure 2. Frequency Error

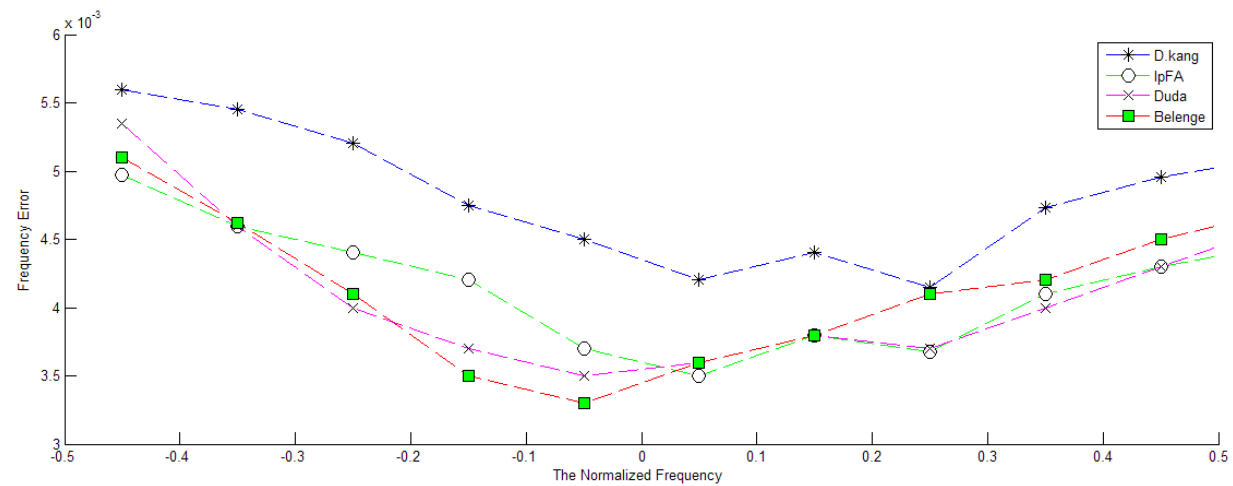

Figure 3. Frequency Error

As illustrated in Figure 2 and Figure 3, the results obtained were not satisfactory for both Duda and Belege being weighted in either the non-Hanning or Hanning windows. For this proposed algorithm, the estimation results obtained from being weighted in non-Hanning window was still quite impressive, where the estimated error was less than. In summary, the above simulation analysis reminded that in the absence of noise or under low-noise conditions, the new algorithm, like the traditional algorithm, is not merely able to obtain better estimates, but also able to be applied to other classic functions in additional to Hanning window.

\section{B. The Study of Ability of Noise Resistance}

In the frequency correction, estimation accuracy in noisy conditions is an important indicator of the algorithm superiority. When noise is present and at a higher level, the signal to noise ratio is allowed to be set to a high value, making it much larger than the threshold, when the traditional methodologies for frequency correction may still estimate the correct spectral line in the wrong position. This is the case in the spectrum correction that is generally referred to as the incorrect polarity estimation (IPE). For example, nearly coherent sampling in 2-point algorithms and the sampling conditions of nearly half of the cycle in 3-point algorithms are prone to the phenomenon of incorrect polarity estimation. The occurrence of IPE phenomenon may seriously affect the signal inversion results and the value of $\hat{\mu}$.

In order to test the new algorithm performance in terms of anti-additive noise, this section made a comparison between the new algorithm and the traditional algorithm. Before that, it was assumed that there was a theoretical signal with additive noise, where SNR was set as $-5 \mathrm{~dB}$, so that the correct polarity estimates may occur (IPE phenomenon). One step was $0.025 \mathrm{hz}$, and the scanning frequency ranged from $255.5 \mathrm{hz}$ to $256.5 \mathrm{hz}$, and the random phase uniformly distributed in $[-\pi, \pi]$. For each frequency, 50,000 separate instances were generated accordingly. Figures 4 shows that the standard deviation error and the mean absolute error of each algorithm on the frequency deviation function in the Hanning windows. 


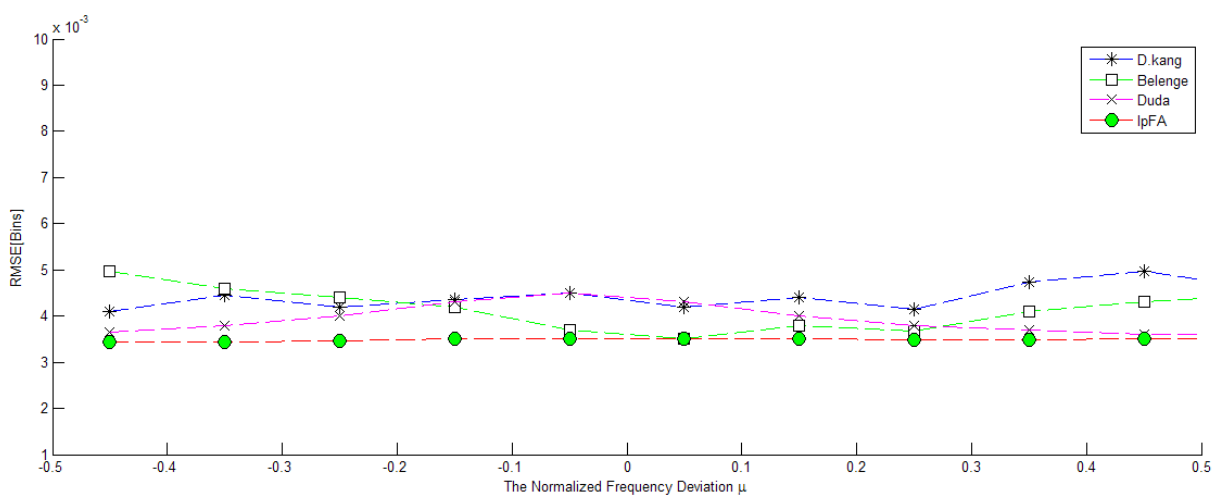

Figure 4. RMSE of different algorithms for the Hanning window (SNR=-5dB)

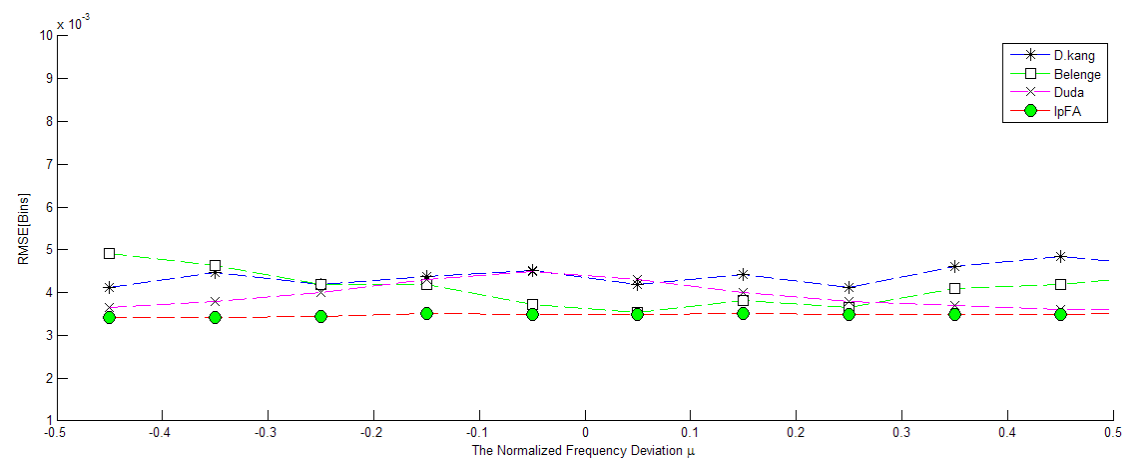

Figure 5. Frequency estimation errors for the Hamming window

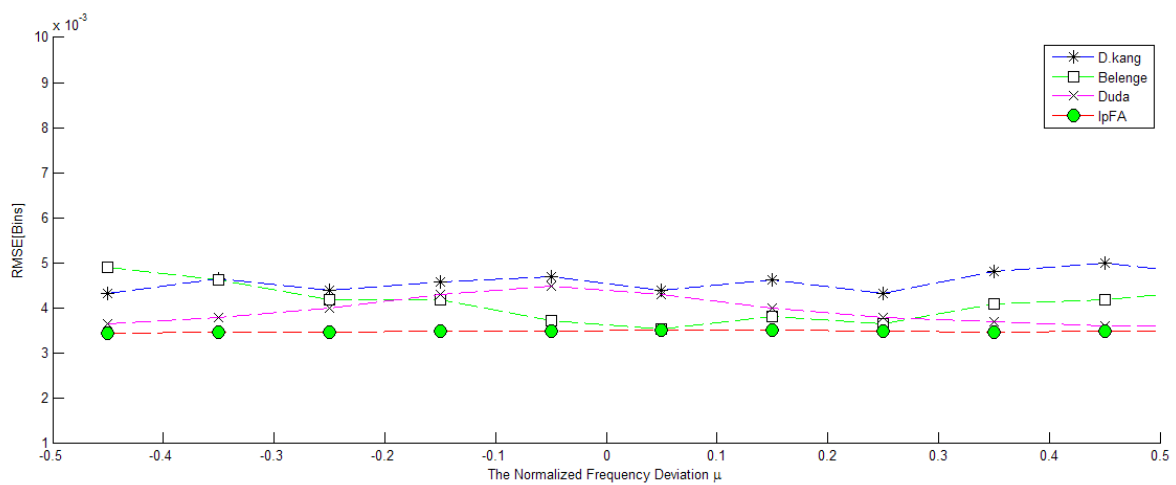

Figure 6. Frequency estimation errors for the Blackman window

Figure 4 shows that estimation accuracy of D.kang is almost the same as Duda's in the Gaussian noise conditions. With an increasing $|\mu|$, the frequency correction errors of these three kinds of algorithms (including standard deviation error and mean absolute error) were increased, respectively. When the value of $\mu$ changed in the range $(-0.5,0.5)$, Belenge had its estimation error in noisy conditions that dropped to a lowest point first, and then continued to rise and reach a higher level. Nonetheless, the proposed algorithm, under similar conditions, had the estimated error values that were almost all equal, except for a few fluctuations, and the most importantly, the smallest error among the estimating results of all. Figures 5 and 6 also exhibit the estimated standard error of Hamming window and Blackman window. Obviously, the results of each method including new algorithms were basically the same as the simulation results with Hanning window in Figure 4. 


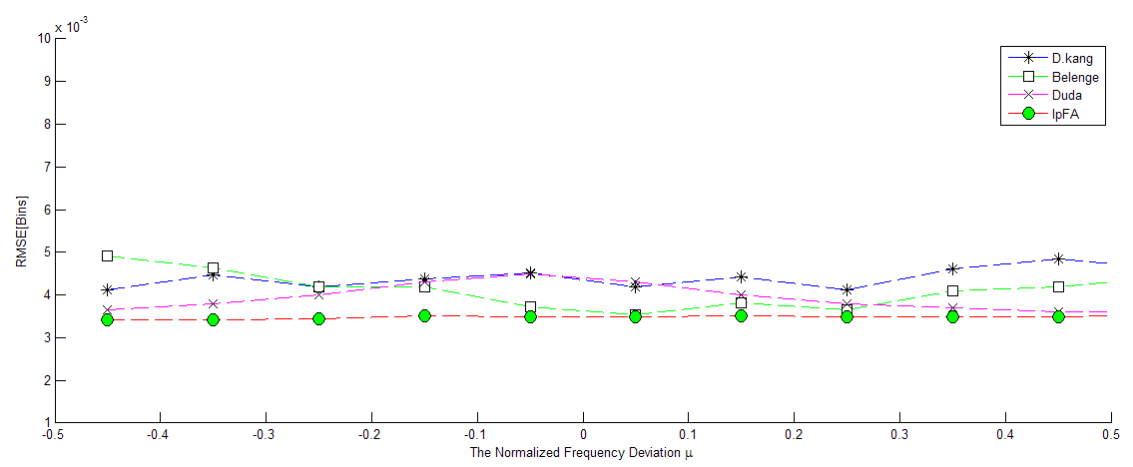

Figure 7. Frequency estimation errors for the Hamming window

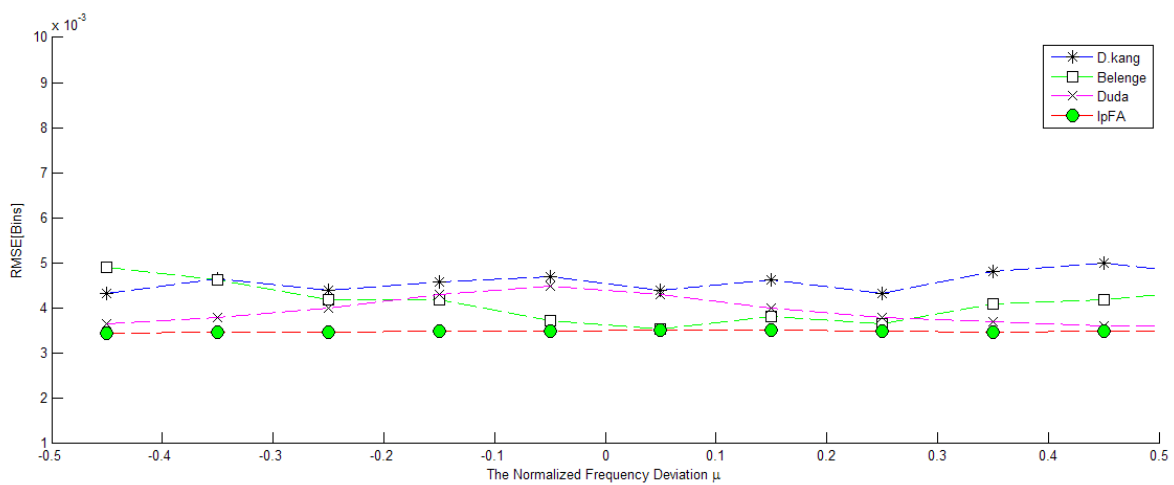

Figure 8. Frequency estimation errors for the Blackman window

In summary, simulation study on different algorithms shows that the traditional methodologies under noisy conditions came out with lower estimation accuracy, compared to the proposed one, which was appealed with the standard deviation of estimation error being lowest in all algorithms, thus demonstrating its high anti-IPE performance. In addition, there are other aspects of advantages that come along with the new algorithm. This is exemplarily evidenced in traditional algorithms, where the line spacing is the frequency resolution, leading to significantly weaken noise correlation. The new algorithm used a more powerful spectrum lines, which features a spectral interval being only $1 / \lambda$ of the frequency resolution. This may contain similar noises in adjacent lines so that it is capable of eliminating most of the noises.

\section{CONCLUSION}

In this paper, an interpolation algorithm has been proposed to provide an accurate estimation of the frequency. The described algorithm is established on the zero padding technique and the main-lobe fitting technique. It is straightforward to understand and possesses the advantage of practical simplicity. The cost is a increase of computation of FFT. The new algorithm is compatible with msot classic windows. It does not need to know the spectrum of the data window, or carry out any calculation in advance. Through computer simulations, the effectiveness of the proposed algorithm for various windows was proved. The influence of systemactic error and white Gaussian noise on the accuracy of frequency estimations was also studied. In addition, the problem of IPE in the traditional interpolation algorithms was discussed. Simulation results show that the new algorithm has a intrinsic robustness against IPE and a higher resistance to additive noise than previous interpolation algorithms.

\section{REFERENCES}

[1] Daniel Belega, Dario Petri . Frequency estimation by two- or threepoint interpolated Fourier algorithms based on cosine windows, Signal Processing.117(2015) 115-125.

[2] Sergio Ledesma, Derong Liu, Donato Hernández. Two approximation methods to synthesize the power spectrum of fractional Gaussian noise, Computational Statistics \& Data Analysis.52(2007) 1047-1062.

[3] Peter Nagel. Homomorphic design of interpolated digital filters and equalisers, Signal Processing.88(2008) 1747-1761.

[4] Chao Gong, Daoxing Guo, Bangning Zhang. Improved Frequency Estimation by Interpolated DFT Method, Procedia Engineering.29(2012) 4112-4116.

[5] M.Xie, K.Ding. Corrections for frequency, amplitude, and phase in a fast Fourier transform of a harmonic signal, Mech. Syst. Signal Process.10(1996)211-221.

[6] Jiufei Luo, Zhijiang Xie, Ming Xie, Frequency estimation of the weighted real tones or resolved multiple tones by iterative interpolation DFT algorithm, Digital Signal Processing.41(2015)118-129.

[7] Miloš Sedláček, Michal Titěra. Interpolations in frequency and time domains used in FFT spectrum analysis, Measurement. 23(1998)185-193. 
[8] Dimitris Kiritsis. High precision interpolation algorithm for 3D parametric curve generation, Computer-Aided Design.26(1994)850-856.

[9] T.Grandke, Interpolation algorithms for discrete Fourier transforms of weighted signals, instrumentation and measurement, IEEE Trans. 32(1983)350-355.

[10] G.Andria,M.Savino,A.Trotta. Windows and interpolation algorithms to improve electric measurement accuracy, instrumentation and measurement, IEEE Trans. 38(1989)856-863.

[11] Daniel Belega. Accuracy analysis of the normalized frequency estimation of a discrete-time sine-wave by the average-based interpolated DFT, Measurement. 46(2013)593-602.

[12] K.Duda. DFT interpolation algorithm for Kaiser-Bessel and DolphChebyshev windows, instrumentation and measurement, IEEE Trans.60(2011)784-790.
[13] D. Belega, D. Dallet. Normalized frequency estimation for accurate dynamic characterization of $\mathrm{A} / \mathrm{D}$ converters by means of the three-parameters sine-fit algorithm, Measurement 41(2008)986-993.

[14] Jiangtao Xi, Joe F. Chicharo. A time-domain interpolation approach for DFT harmonic analysis, Signal Proces. 58(1997)181192.

[15] Kui Fu Chen, Yan Feng Li. Combining the Hanning windowed interpolated FFT in both directions, Computer Physics Communications. 178(2008)924-928.

[16] Kui Fu Chen, Xu Cao, Yang Feng Li. Sine wave fitting to short records initialized with the frequency retrieved from Hanning windowed FFT spectrum, Measurement. 42(2009)127-135. 